\title{
Florid Cemento-Osseous Dysplasia Masquerading as Osteomyelitis: An Exceptional Case Report in an Indian Female
}

\author{
Harveen Kaur ${ }^{1}$ Deepa Jatti Patil ${ }^{2}$ \\ ${ }^{1}$ Department of Oral Medicine \& Radiology, Dr Harvansh Singh \\ Judge Institute of Dental Sciences \& Hospital, Panjab University, \\ Chandigarh, India \\ 2Department of Oral Medicine \& Radiology, KM Shah Dental College \\ \& Hospital, Sumandeep Vidyapeeth, Vadodara, Gujarat, India
}

\begin{abstract}
Address for correspondence Harveen Kaur, BDS, MDS, Department of Oral Medicine \& Radiology, Dr Harvansh Singh Judge Institute of Dental Sciences \& Hospital, Panjab University, E1-95, Panjab University Campus, Sector 14, Chandigarh 160014, India (e-mail: drharveenkaur.pu@gmail.com).
\end{abstract}

\author{
Abstract \\ Keywords \\ - florid cemento-osse- \\ ous dysplasia \\ - fibro-osseous lesions \\ - multiple \\ radio-opacities
}

Florid cemento-osseous dysplasia consists of a group of fibro-osseous lesions with multiquadrant involvement, generally seen in the tooth-bearing portions of the mandible. These lesions are most common in middle-aged black women and rare in the Indian population, with only five cases been documented in the international literature in the Indian population. This manuscript presents a case of a 60 -year-old female patient, who presented with a history of nonhealing extraction socket for 2 years and on examination demonstrated ulcerated alveolar mucosa with exposed necrotic bone. Panoramic and computed tomography images revealed multiple homogenous radio-opacities throughout the jaws. The lesion was diagnosed as florid cemento-osseous dysplasia on the basis of clinical and radiologic examination. The patient was prescribed antibiotics with iodoform dressings and proper oral hygiene maintenance.

\section{Introduction}

Florid cemento-osseous dysplasia (FCOD) refers to a group of fibro-osseous lesions, involving more than one quadrant of the oral cavity. ${ }^{1}$ The term FCOD was proposed in the second edition of the World Health Organization's (WHO) International Histological Classification of Tumors. ${ }^{2}$ It is described as "Lobulated masses of dense, highly mineralized, almost acellular cement-osseous tissue typically occurring in several parts of the jaw."2 FCOD is a rare condition of the jaws, commonly prevalent in middle-aged black women, but may also be seen in Caucasians and Asians.,

The etiology of FCOD is unknown but is thought to be associated with reactive or dysplastic processes originating from elements of the periodontal ligaments. ${ }^{5-8}$ Although the lesions are usually asymptomatic, but they may be discovered during routine radiographic examinations, presenting themselves as multiple radiopaque sclerotic masses surrounded by a peripheral radiolucent rim, with the most common location being the tooth-bearing regions of the oral cavity. ${ }^{9}$ Generally, the lesions do not require any treatment, but due to the avascularity of the lesions, there is increased susceptibility to osteomyelitis due to secondary infection after trauma. ${ }^{5,8}$ This article describes a rare case of FCOD in an Indian female, who reported with a nonhealing extraction socket since 2 years, and was diagnosed on the basis of clinical and radiographic findings.

\section{Case Report}

A 60-year-old Indian female reported with a chief complaint of dull, continuous pain in a nonhealing extraction socket since she got her lower left posterior teeth extracted due to caries 2 years back. Her medical and family history was noncontributory. On intraoral examination, a large area of ulcerated alveolar mucosa with exposed, necrotic, tender bone was seen in the lower left posterior region of the mandible, without any pus discharge, sinus or fetid odor, expansion of the mandible, paresthesia/hypoesthesia ( - Fig. 1). Bilateral submandibular lymph nodes were palpable, mobile, tender, and a provisional diagnosis of chronic nonsuppurative osteomyelitis was given, based on the clinical findings. published online November 26, 2019
DOI https://doi.org/

10.1055/s-0039-3400342 ISSN 2321-1482.
(C)2019 Bhojia Dental College and Hospital affiliated to Himachal Pradesh University
License terms

(ㅇ) (1) $\ominus \circledast$ 


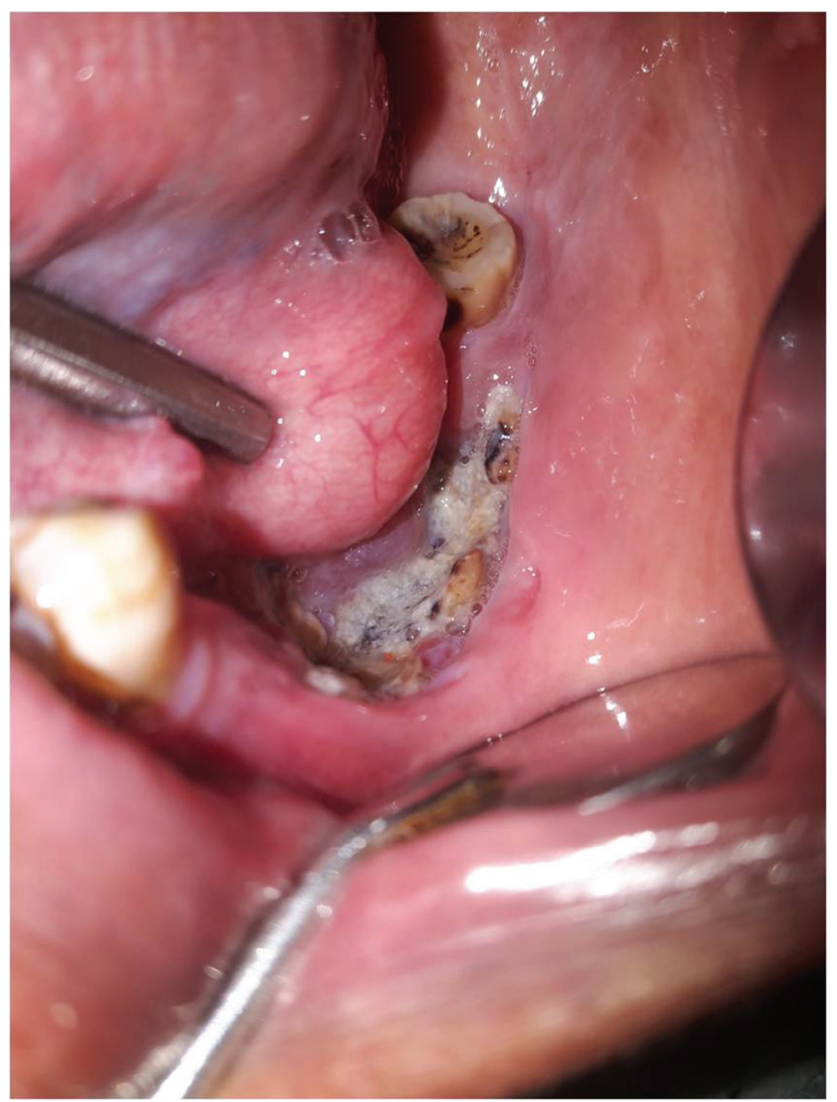

Fig. 1 Nonhealing extraction socket in the left posterior region of the mandible and ulcerated alveolar mucosa with exposed, necrotic bone.

Panoramic radiograph revealed a diffuse irregular area of moth-eaten radiolucency interspersed with radio-opacity of the crest of the alveolar ridge in the left mandibular posterior region above the inferior alveolar canal and multiple homogeneous sclerotic masses with radiolucent rims in the tooth-bearing regions of the mandible and maxilla, bilaterally, above the inferior alveolar canal (-Fig. 2). The ramus, angle, and the body of mandible were spared. Full mouth intraoral periapical radiographs revealed hypercementosis with respect to $15,17,28,44,45,47$, lower anteriors and root clubbing in 12, 22 and maxillary left posterior teeth. Computed tomographic (CT) images of mandible and maxilla revealed multiple radio-opacities throughout the jaw. Axial

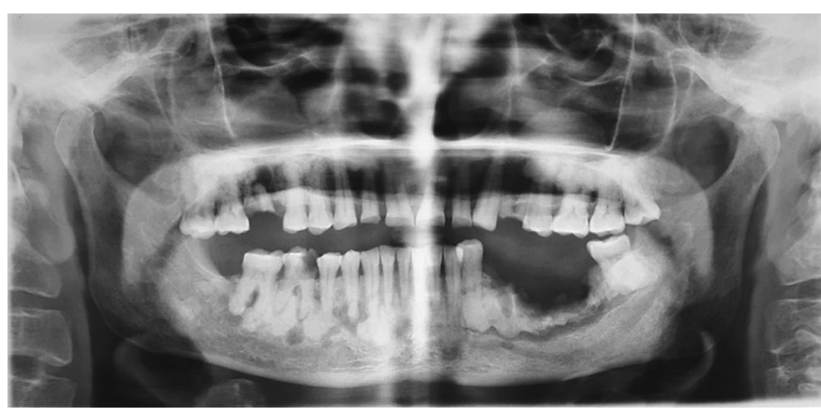

Fig. 2 Panoramic radiograph revealing generalized multiple homogenous radio-opacities in the tooth-bearing regions of jaw with radiolucent rims, inferiorly displaced mandibular canal. view of the mandible in the left posterior region also revealed irregular radiolucency interspersed with radio-opacity with discontinuity of the buccal cortical plate suggestive of osteomyelitis. Skeletal survey did not reveal similar lesions in the other parts of the body. Based on the radiographic features, a differential diagnosis of FCOD, Paget's disease, and fibrous dysplasia (FD) was considered.

Biochemical analyses including complete blood count, fasting blood glucose, serum calcium, phosphorus, and alkaline phosphatase levels were performed to rule out Paget's disease and FD. All the laboratory investigations were within normal limits.

Based on the clinical, radiological, and biochemical analysis, a final diagnosis of FCOD was given and the patient was put on clindamycin $600 \mathrm{mg}$ thrice daily for 1 week along with iodoform dressings and proper oral hygiene maintenance. When she was recalled for follow-up after few days, she showed marked improvement.

\section{Discussion}

Cemento-osseous dysplasias include a group of disorders with a similar pathogenesis and are believed to originate from periodontal ligament tissues. The classification of these lesions, by the WHO (1992), was based on age, sex, location, histopathologic, radiographic, and clinical characteristics of the lesions, and included cemento-ossifying fibroma, benign cementoblastoma, and the cemento-osseous dysplasia group (periapical cemental dysplasia and FCOD). ${ }^{10}$ Presently, based on their location and radiographic appearances, they are classified into three main groups: periapical (periapical regions, bilaterally), florid (sclerotic symmetrical masses in the maxilla/mandible), and focal (single lesion) cemental dysplasias. ${ }^{11}$

FCOD is generally seen as a non-neoplastic, reactive process in the tooth-bearing regions of the jaws, most frequently in middle-aged black women (African origin). ${ }^{4}$ It is more common in the middle-aged group, black females, them being two times more commonly affected than the males. ${ }^{12,13}$

There is a great dilemma regarding the pathogenesis of the FCOD. One school of thought believes it to be due to the proliferation of the fibroblastic mesenchymal stem cells in the periodontal ligament, while another says that the remnants of the cementum, after tooth extraction, lead to the formation of the FCOD lesions, yet others believe that the lesions are basically reactive or dysplastic changes originating from the periodontal ligaments.7.8

FCOD is generally asymptomatic, but exposure of FCOD lesions in the oral cavity may lead to symptoms, ranging from dull pain to drainage. ${ }^{13}$ The lack of symptoms leads to this lesion being identified through incidental clinical or radiological findings, as was seen in the present case, when the patient reported with osteomyelitis, which generally is the most common complication in FCOD due to avascularity of the lesion.

Radiographically, the lesions may be radiolucent, radiodense or mixed, depending on the stage and may involve more than one tooth-bearing regions of the oral cavity. The 
lesion is usually located in the periapical region of the teeth, but may expand in any direction depending upon the aggressiveness of the lesion. Initially, the lesion appears as a radiolucent one, further giving rise to a mixed stage and finally to a radio-opaque stage. ${ }^{8}$ The lesion characteristically appears as diffuse, lobular, irregularly shaped radio-opacities of the mandible and/or maxilla, as was also seen in the panoramic images of the present case.

Axial CT images in the present case clearly showed the location and extent of the lesion, especially the bilateral cortical bone expansion in the posterior maxilla. CT can be used to differentiate FCOD from lesions that exhibit a similar sclerotic appearance on conventional radiographs.

Histopathological examination reveals cellular fibrous tissue, containing trabeculae of woven bone with cementum-like calcification in the early stages and curvilinear trabeculae with increased mineralization in the mature radio-opaque stage. ${ }^{12}$ Biopsy is often avoided due to the acellular and avascular nature of the lesion and also due to the increased risk of osteomyelitis in FCOD. The diagnosis of FCOD is usually clinical and radiographic only with histological investigation not being necessary, according to Jerjes et al. ${ }^{11}$ Hence, like many other researchers the present case was also diagnosed as FCOD on the basis of clinical and radiographic findings and no biopsy was done.

FCOD should be differentiated from Paget's disease, chronic diffuse osteomyelitis, osseous dysplasias (OD), ossifying fibroma (OF), FD and Gardner's syndrome. FCOD has no other skeletal changes, skin tumors, or dental anomalies as seen in Gardner's syndrome. Unlike FCOD, Paget's disease is often polyostotic and elevated alkaline phosphate levels are seen in the serum. ${ }^{14,15}$

Lesions of chronic diffuse sclerosing osteomyelitis appear as a single, poorly delineated opaque segment of the mandible, whereas lesions of the FCOD are seen as multiple round opaque masses, confined to tooth-bearing areas. ${ }^{10}$

Jaw expansion is mostly not seen in OD with the exception of familial gigantiform cementoma that occurs at a young age, in Caucasians with an autosomal dominant inheritance trait. ${ }^{5,7,8}$ FCOD may be familial and in rare cases may exhibit an autosomal-dominant inheritance pattern.,10 OF is generally seen in the females, posterior mandible, and in second to fourth decades of life. ${ }^{13}$ Clinical features of FD include facial asymmetry, irregular cafe-au-lait spots, displacement of teeth and malocclusion, which are not seen in FCOD.

Management of FCOD patients is difficult due to the increased risk of development of chronic inflammation and infection within the densely mineralized tissue. Sequestration of cementum like masses occurs slowly, often followed by healing. Presence of symptoms indicates inflammation (chronic osteomyelitis); hence, administration of antibiotics is advised. ${ }^{11}$ Martini prescribed clindamycin at 600 mg every 6 hours, which is well distributed in the osseous tissue. ${ }^{6}$ However, response to antibiotics may be dubious, owing to the decreased vascularity of the lesion. ${ }^{8}$ Some researchers recommend to avoid extraction in patients with FCOD, due to the increased risk of osteomyelitis after trauma.,
The last option in the management of FCOD includes surgical removal of the sclerotic masses and sequestrum, with proper and regular patient follow-up (clinical and radiographic examination) with good oral hygiene maintenance. ${ }^{5,6,13}$ After thorough analysis of cases reported worldwide, this is the sixth case of FCOD, of Indian origin reported in the International literature, making it a rare occurrence in Indians. Diagnosis of FCOD is based on the clinical and radiographic examination; hence, dentists play an important role in identifying such lesions, paving a way for proper diagnosis, treatment, and planning.

\section{Conflict of Interest}

None declared.

\section{References}

1 Ong ST, Siar CH. Florid cemento-osseous dysplasia in a young Chinese man. Case report. Aust Dent J 1997;42(6):404-408

2 Kramer IRH, Pindborg JJ, Shear M. Histological Typing of Odontogenic Tumours. World Health Organisation, International Histological Classification of Tumours. 2nd edition. Berlin, Germany: Springer-Verlag; 1992 29-30

3 Miyake M, Nagahata S. Florid cemento-osseous dysplasia. Report of a case. Int J Oral Maxillofac Surg 1999;28(1):56-57

4 Waldron CA. Fibro-osseous lesions of the jaws. J Oral Maxillofac Surg 1985;43(4):249-262

5 Sarmento DJ, Monteiro BV, de Medeiros AM, da Silveira EJ. Severe florid cemento-osseous dysplasia: a case report treated conservatively and literature review. Oral Maxillofac Surg 2013;17(1):43-46

6 Martini MZ, de Carvalho Júnior JP, Soares HA. Surgical management of an infected aggressive florid cemento-osseous dysplasia. Report of a case. Minerva Stomatol 2006;55(9):515-521

7 Coleman H, Altini M, Kieser J, Nissenbaum M. Familial florid cemento-osseous dysplasia-a case report and review of the literature. J Dent Assoc S Afr 1996;51(12):766-770

8 Bencharit S, Schardt-Sacco D, Zuniga JR. Minsley GE. Surgical and prosthodontic rehabilitation for a patient with aggressive florid cemento-osseous dysplasia: a clinical report. J Prosthet Dent 2003;90(3):220-224

9 Babaria U, Patel H, Pandya $\mathrm{H}$, et al. Florid osseous dysplasia: a case report. J Dent Sci 2011;2:10-11

10 Gonçalves M, Píspico R, Alves FdeA, Lugão CE, Gonçalves A. Clinical, radiographic, biochemical and histological findings of florid cemento-osseous dysplasia and report of a case. Braz Dent J 2005;16(3):247-250

11 Jerjes W, Banu B, Swinson B, Hopper C. Florid cemento-osseous dysplasia in a young Indian woman. A case report. Br Dent J 2005;198(8):477-478

12 Sanjai K, Kumarswamy J, Kumar VK, Patil A. Florid cemento osseous dysplasia in association with dentigerous cyst. J Oral Maxillofac Pathol 2010;14(2):63-68

13 Gündüz K, Avsever H, Karaçayli U, Senel B, Pişkin B. Florid cemento-osseous dysplasia: a case report. Braz Dent J 2009;20(4):347-350

14 White SC, Pharoah MJ. Oral Radiology - Principles and Interpretation. 4th edition. Saint Louis: Mosby; 2000

15 Wolf J, Hietanen J, Sane J. Florid cemento-osseous dysplasia (gigantiform cementoma) in a Caucasian woman. Br J Oral Maxillofac Surg 1989;27(1):46-52 